\title{
Heliospheric current sheet inclinations at Venus and Earth
}

\author{
G. Ma, K. Marubashi, and T. Maruyama \\ Communications Research Laboratory, 4-2-1 Nukui-Kitamachi Koganei-shi Tokyo, Japan
}

Received: 25 May 1998 / Revised: 16 September 1998 / Accepted: 22 October 1998

\begin{abstract}
We investigate the inclinations of heliospheric current sheet at two sites in interplanetary space, which are generated from the same solar source. From the data of solar wind magnetic fields observed at Venus $(0.72$ AU) and Earth (1 AU) during December 1978-May 1982 including the solar maximum of 1981,54 pairs of candidate sector boundary crossings are picked out, of which 16 pairs are identified as sector boundaries. Of the remainder, 12 pairs are transient structures both at Venus and Earth, and 14 pairs are sector boundaries at one site and have transient structures at the other site. It implies that transient structures were often ejected from the coronal streamer belt around the solar maximum. For the 16 pairs of selected sector boundaries, we determine their normals by using minimum variance analysis. It is found that most of the normal azimuthal angles are distributed between the radial direction and the direction perpendicular to the spiral direction both at Venus and Earth. The normal elevations tend to be smaller than $\sim 45^{\circ}$ with respect to the solar equatorial plane, indicating high inclinations of the heliospheric current sheet, in particular at Earth. The larger scatter in the azimuth and elevation of normals at Venus than at Earth suggests stronger effects of the small-scale structures on the current sheet at $0.72 \mathrm{AU}$ than at $1 \mathrm{AU}$. When the longitude difference between Venus and Earth is small $\left(<40^{\circ}\right.$ longitudinally), similar or the same inclinations are generally observed, especially for the sector boundaries without small-scale structures. This implies that the heliospheric current sheet inclination tends to be maintained during propagation of the solar wind from $0.72 \mathrm{AU}$ to $1 \mathrm{AU}$. Detailed case studies reveal that the dynamic nature of helmet streamers causes variations of the sector boundary structure.
\end{abstract}

Key words. Interplanetary physics (interplanetary magnetic fields; sources of solar wind)

Correspondence to: G. Ma; e-mail: ma@crl.go.jp

\section{Introduction}

It has been generally accepted that at large heliocentric distances the heliospheric current sheet is a warped surface separating regions of oppositely directed interplanetary magnetic fields (IMFs) rooted at the sun (Schulz, 1973; Alfvén, 1977). Inclination is one of the basic parameters describing the extent of the warp of the heliospheric current sheet. Many authors attempted to deduce the sheet inclination by applying minimum variance analysis (MVA) (Sonnerup and Cahill, 1967) to single spacecraft IMF observations though no uniform definition of the current sheet and definite rule for selecting the time interval over which to perform the minimum variance analysis exist yet. A heliospheric current sheet or a sector boundary is generally a transition region between two sectors. Each sector is a nearly unipolar region in which the IMF is close to the spiral direction. Klein and Burlaga (1980) stated that each sector should persist for at least two days. The transition should occur in less than two days. They treated the multiple discontinuities crossing as a "thick" sheet ( $\geq 3 \mathrm{~h}$ ), and excluded intervals containing transient events. Villante and Bruno (1982) studied well-defined, thin current sheets $(\leq 1 \mathrm{~h})$ for three solar rotations. The shortest interval between two current sheets that they analyzed was $32 \mathrm{~min}$.

Using K-coronameter observations, Behannon et al. (1983) considered two cases of heliospheric current sheet crossings observed by Voyager 1 and 2. One case was a vertical current sheet and the other was a horizontal current sheet with respect to the solar equatorial plane estimated by coronameter data. For the vertical sheet crossing at $2.8 \mathrm{AU}$, minimum variance analysis based on hourly averaged IMF data gave a normal with an elevation of $6^{\circ}$. The other case was of horizontal multiple crossings at $1.4 \mathrm{AU}$. The minimum variance analysis of individual sheets with 1.92-s average data did not give normals highly inclined out of the solar 
equatorial plane. They concluded that the minimum variance direction would be quite different from the normal to the current sheet when small-scale variations existed within or near the transition region. Suess (1984), however, pointed out that the current sheet inclination could vary with heliocentric distances when the solar wind velocity gradient was large.

Based on previous results of observational studies, Crooker et al. (1993) proposed a multiple helmets model which implies that the heliospheric current sheet is not a single surface but a constantly changing layer with a varying number of current sheets of finite extent filling the finite thickness of the coronal streamer belt. They proposed that the coronal streamer belt behaves as a conduit for outflow from quiet and transient ejections. A multiple current sheets structure stems from the multiple helmets which generate small-scale ejections. For an example of multiple current sheets crossing, Crooker et al. (1993) showed that minimum variance normals are different for each sheet, the same as in Behannon et al. (1983). By applying the minimum variance analysis to the entire transition region, however, they got a normal which was exactly orthogonal to the sector boundary derived from source surface model calculations made by Hoeksema and Scherrer (1986). To test the validity of the source surface model as a predictor of the heliospheric current sheet orientation, Burton et al. (1994) recently compared the model with the minimum variance determination of the inclination from the IMF data. They selected the time interval as the entire interval which contained alternating or ambiguous polarity. Their conclusions were that the source surface model was an excellent predictor of the inclination of the heliospheric current sheet without transient events at 1 AU.

Measuring the current sheet inclination at two sites in interplanetary space would give us a better identification and description of sector boundaries. In this paper we examine the effects of interplanetary dynamics on the inclinations of the heliospheric current sheets at different latitudes and longitudes by comparing the IMF data obtained from the Pioneer Venus orbiter (PVO) (Russell et al., 1980) with those from near-Earth observations. The near-Earth data were taken from the OMNI data tape (Couzens and King, 1986). Our study concentrates on a time interval 1979-1982 when the current sheet was most disordered and inclined out of the equatorial plane, which includes the solar maximum of 1981 (e.g. Suess et al., 1993). We start by selecting pairs of sector boundaries at Venus and Earth originating from the same solar longitude. Then we estimate the inclination of the sector boundaries for each pair by using minimum variance analysis. The effects of the coronal streamer belt dynamics on sector boundaries are discussed in detail by two case studies.

\section{Sector boundary identification}

A sector boundary is defined by a transition region between opposite sectors. The transition from one sector to another should take less than two days. Each sector should persist for at least two days (Klein and Burlaga, 1980). The sector boundary is usually thin so that the IMF goes rapidly from one stable direction to another within several hours. When the boundary is thick, the IMF changes direction gradually over a two-day period. Sometimes the boundary can be a region of multiple current sheet crossings. In this study, we require that the IMFs are parallel to each other for at least $3 \mathrm{~h}$ just before and right after the transition. Transient structures are not taken into account.

According to the above definition, we identify a sector boundary in two steps. First we look for transition region between opposite sectors, where each sector persists for at least two days with the same polarity, and the transition from one sector to the other takes less than two days. The second step is to examine that in every sector the IMFs are parallel to each other for at least $3 \mathrm{~h}$ just before and soon after the transition.

We define IMF polarities as positive $(+)$ or negative $(-)$ when the IMF's azimuthal angle falls within $\pm 40^{\circ}$ of the outward or the inward spiral direction. If the IMF vector falls outside this limit, the polarity is considered ambiguous. For 1-h average IMF data in Venus solar orbital (VSO) and geocentric solar ecliptic (GSE) coordinate systems, we count the number of positive, negative and ambiguous polarities for each day to determine the most frequent polarity for that day. Assuming 5-day and 4-day propagation times from the sun to Earth and to Venus, respectively, we map the daily averages of IMF polarities back to the sun's surface and make a Carrington diagram of the IMF polarities, as shown in Fig. 1. We use a diamond $(\diamond)$ to show ambiguous polarity, and a triangle $(\triangle)$ to indicate gap in the data in the figure. A candidate sector boundary is identified by the transition between two sectors, where each sector persists for at least two days with the same polarity. If there are ambiguous polarities, the transition from one sector to another should take less than two days. We then obtain a candidate heliospheric current sheet pair generated from the same heliographic longitude on the sun within one Carrington rotation (CR).

Figure 1 shows diagrams of the IMF polarities for CR1703 and CR1704. Thick represents observations from near-Earth spacecraft, and thin represents observations from PVO. The dates followed by "(VENUS)" shown in the figure are for the PVO data. The dates followed by "(EARTH)" are for the near-Earth data. General agreement is found between PVO and nearEarth polarities. Six pairs of transitions are indicated with marks from T1 to T6. Three pairs are selected as candidate sector boundaries, which are shown with arrows. We do not select the transitions T3 and T4 because negative polarities at Venus do not persist for two days. We discard T6 for the same reason of negative polarities at Earth. Actually T6 was identified as a pair of flux ropes by Marubashi (1996).

Forty Carrington rotations are included in this study. Fifty-four pairs of candidate sector boundary crossings are confidently picked out. These pairs of 

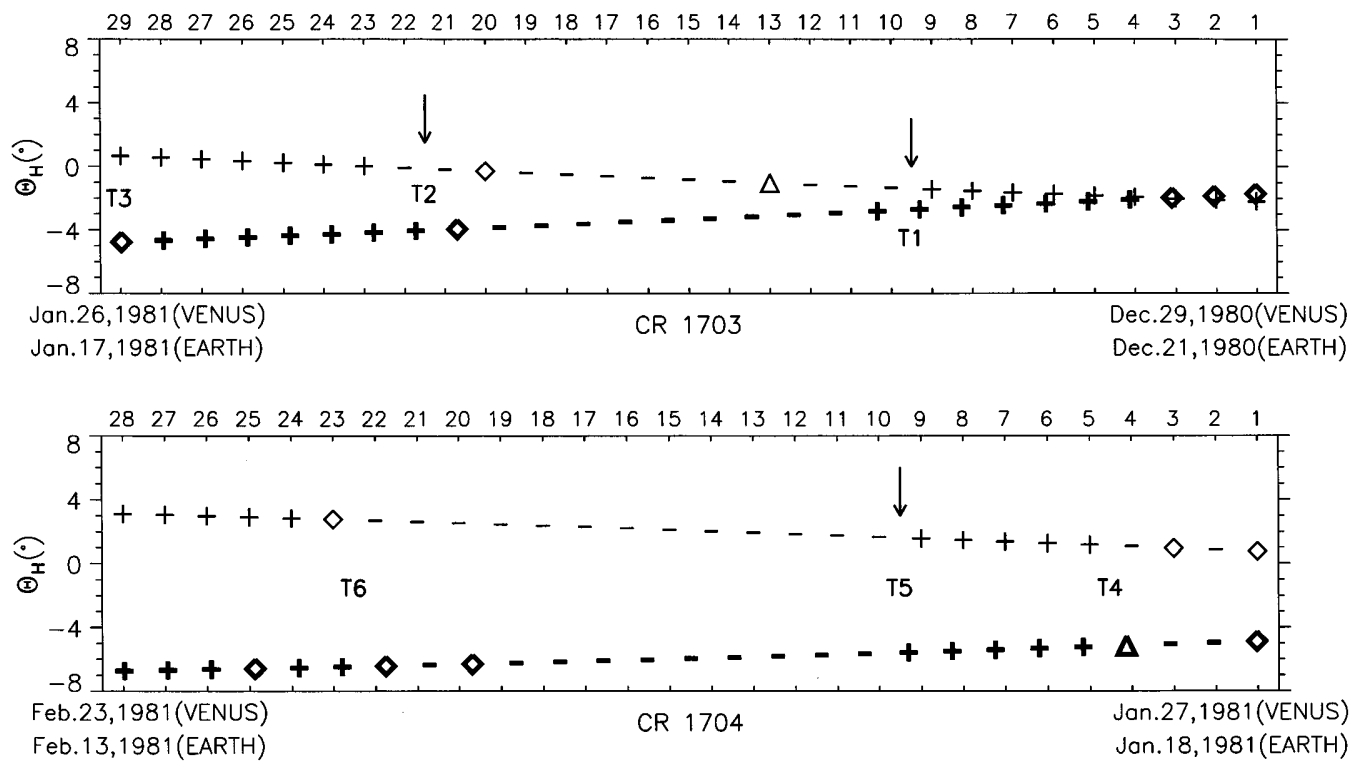

Fig. 1. Daily averages of the IMF polarity observed at Venus (thin) and Earth (thick) for Carrington rotation (CR) 1703 (upper panel) and 1704 (lower panel), adjusted for the 4-day and 5-day transit times of

boundaries agree well in locations with those calculated from the source surface model (Hoeksema and Scherrer, 1986).

We examine whether a candidate sector boundary satisfies the criterion of the second step by using the plots of the magnetic field vectors projected on the $\mathrm{X}-\mathrm{Y}$, $\mathrm{X}-\mathrm{Z}$ and $\mathrm{Y}-\mathrm{Z}$ planes of VSO or GSE. If the IMF vectors projected on the $\mathrm{X}-\mathrm{Y}$ plane are parallel to each other and persist for at least three hours just before and after the transition, and IMF magnitudes are in general level, this case would be selected as a sector boundary. Although recently there have been some questions as to whether field direction alone can be used to identify true sector boundaries (Kahler and Lin, 1995), we should be able to avoid misidentifying a sector boundary to some extent by comparing the IMF observations at the Venus orbit and the Earth orbit.

If there are transient signatures within the transition region of the candidate sector boundary, such an event would not be taken as a sector boundary. Here, only large-scale transient structures such as flux ropes or shocks are excluded. A flux rope is identified on the basis of the following characteristics: (1) the magnetic field vector rotates smoothly in the $\mathrm{Y}-\mathrm{Z}$ plane by about $180^{\circ}$; (2) the magnetic field strength is higher than background level (refer to Klein and Burlaga, 1982).

From the 54 pairs of candidate sector boundaries, 16 pairs of heliospheric current sheets are selected. The remaining 36 pairs include 4 pairs of sector boundaries with transitions longer than two days; 8 pairs of sector boundaries with data gaps within transition region; 14 pairs of sector boundaries at one site and transient structures at the other; and 12 pairs of transient structures. The results imply that sector boundary is much more dynamic in nature than widely accepted hitherto. Recent observations by $\mathrm{SOHO} / \mathrm{LASCO}$ show solar wind from the sun to Venus and Earth, respectively. The numbers above the frame show the day for the PVO data within one Carrington rotation. See text for detailed explanation

that there are continual transient releases of mass from streamers (Howard et al., 1997; Sheeley et al., 1997). The fact that transient structures are often observed at sector boundary crossings can be interpreted by the result of the transient outflows ejected from the solar streamer belt (see also Crooker et al., 1993).

\section{Sector boundary inclinations at Venus and Earth}

We determine the inclinations of the sector boundaries of the selected 16 pairs by applying minimum variance analysis to the IMFs of the transition region. When performing minimum variance analysis, the time interval over which the minimum variance analysis is applied should include the whole transition region. Based on the plot of IMF vectors projected on the X-Y plane of VSO or GSE, we choose the start time to be the time corresponding to the second parallel vector before the transition, and the end time to be the time corresponding to the second parallel vector after the transition. In addition, the ratio of intermediate eigenvalue $\left(\lambda_{\mathrm{d}}\right)$ to minimum eigenvalue $\left(\lambda_{\mathrm{n}}\right)$ from minimum variance analysis is a measure of how well the normal to the heliospheric current sheet is determined. A value greater than two is considered acceptable (Lepping and Behannon, 1980). For one case among these 16 pairs, the ratio $\lambda_{\mathrm{d}} / \lambda_{\mathrm{n}}$ at Venus is 1.8. Because 1.8 is the limit value (Lepping and Behannon, 1980), and the corresponding sector boundary at Earth is the only one with a normal highly inclined to the equatorial plane among the selected sector boundaries at Earth studied here, we keep this pair for analysis. The ratio is greater than three for 11 sector boundaries at Venus, and for 15 at Earth.

Results of our analysis of the inclinations are summarized in Table 1 for the 16 sector boundary pairs. The table lists interval, which gives the start time 
Table 1. Sector boundaries observed at Venus and Earth; * time lag is not estimated because of the small-scale structures contained within one or both sector boundaries

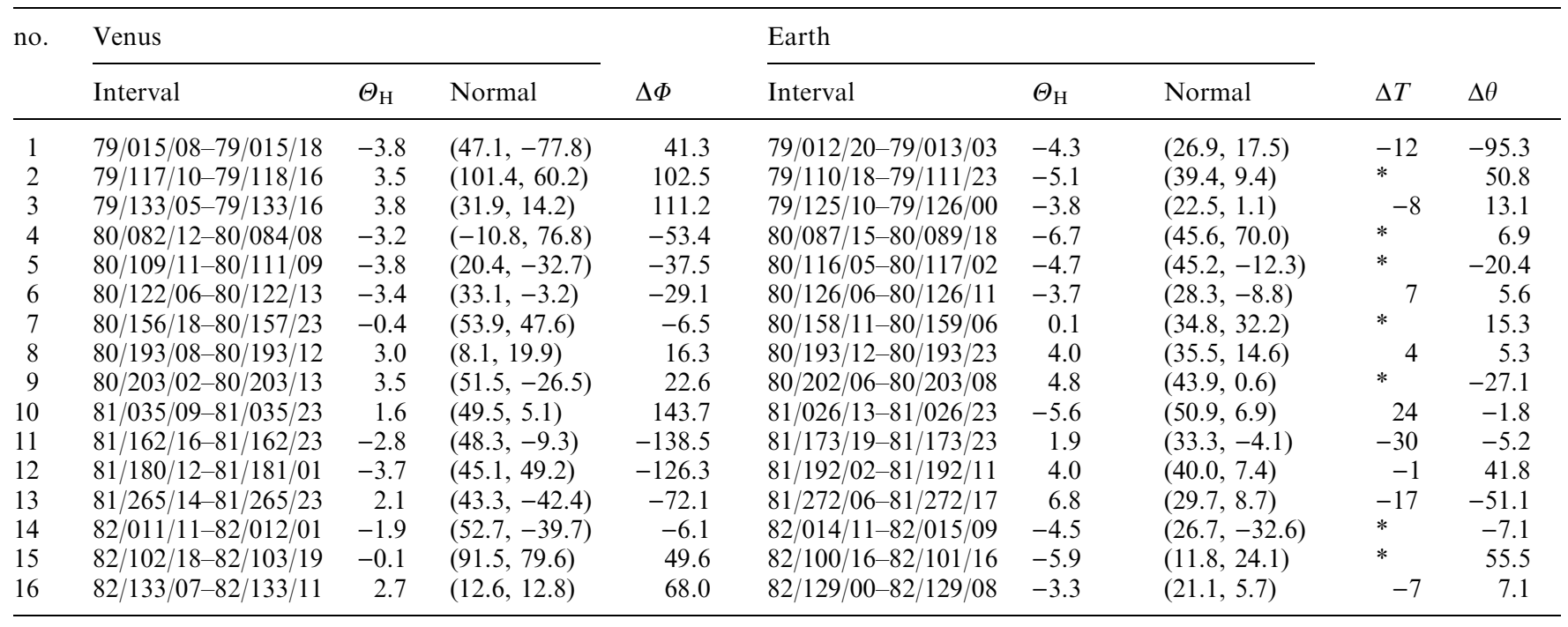

and the end time of a sector boundary crossing for minimum variance analysis, the heliographic latitude $\left(\Theta_{\mathrm{H}}\right)$ of Venus and Earth when the sector boundary crosses them, and the normal to the sector boundaries. The normals are expressed in azimuthal and elevation angles $(\phi, \theta)$ in a coordinate system, in which the $\mathrm{X}$ and $\mathrm{Y}$ axes lie in the solar equatorial plane, $\mathrm{X}$ is the radial line and is directed toward the sun, $\mathrm{Y}$ is the tangent of the equatorial circle and points against the rotation of the sun, and $\mathrm{Z}$ is the vector product of $\mathrm{X}$ and $\mathrm{Y}$. As the normals $(\phi, \theta)$ and $\left(180^{\circ}+\phi,-\theta\right)$ are identical, we selected normal vectors so that their $\phi$ is within $45^{\circ} \pm 90^{\circ}$. The right-hand two columns in Table 1 give results of a comparison of the sector boundaries at Venus and Earth. $\Delta \theta$ is the elevation difference of normals of a pair. The time lag $\Delta T$ is the difference between the time ( $\left.t_{\text {Eobs }}\right)$ when the sector boundary is observed at the Earth orbit and the time $\left(t_{\text {Ecal }}\right)$ estimated from the Venus observation, taking into account the effect of the sun's rotation and the propagation time that the solar wind takes to travel from Venus to Earth. It is expressed by,

$\Delta T=t_{\text {Eobs }}-t_{\text {Ecal }}$

$t_{\mathrm{Ecal}}=t_{\mathrm{Vobs}}-\Delta \Phi / \Omega+\Delta \mathrm{R} / \mathrm{V}_{\mathrm{sw}}$,

where $t_{\text {Vobs }}$ means the time when the sector boundary is observed at the Venus orbit. $\Delta \Phi$ is the Carrington longitudinal difference between Venus and Earth when the sector boundary crosses Venus, which is also listed in Table $1 . \Omega$ is the angular velocity of solar rotation as viewed from Venus, $\Delta \mathrm{R}$ is the distance from 0.72 to $1 \mathrm{AU}$, and $\mathrm{V}_{\mathrm{sw}}$ is the solar wind speed. Here the solar wind speeds are available only for the near-Earth data, we assume that the solar wind speed at Venus was the same as that at Earth when the time lag $\Delta T$ was calculated. A positive value of $\Delta T$ indicates that the sector boundary crossed Earth later than is calculated, while a negative value indicates that the sector boundary crossed Earth earlier than estimated. If the transition region contains ambiguous or mixed polarities and is longer than $3 \mathrm{~h}$, it is difficult to determine a specific time when the sector boundary is crossed. So we do not estimate the time lag.

Figure 2 shows the normal's azimuthal and elevation angles for the 16 pairs of sector boundaries at Venus and Earth separately. According to Parker spiral model, the
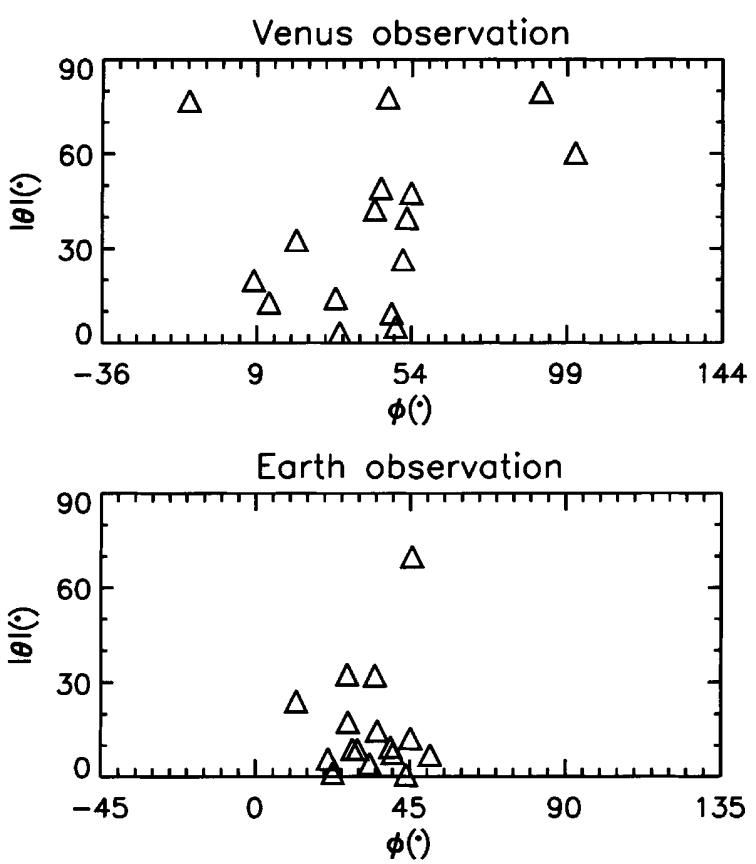

Fig. 2. Normal's azimuth $(\phi)$ and elevation $(\theta)$ for sector boundaries at Venus (upper panel) and Earth (lower panel). The normals tend to be lying between the radial direction and the direction perpendicular to the spiral direction and close to $\left(\leq 45^{\circ}\right)$ the solar equatorial plane, especially at Earth 
spiral angle is $135^{\circ}$ at Earth and $126^{\circ}$ at Venus azimuthally. Since previous studies show that, normals to the heliospheric current sheets tend to be perpendicular to the spiral (Klein and Burlaga, 1980; Burton et al., 1994;), we set $54^{\circ}$ and $45^{\circ}$ respectively to be the centers of the horizontal axis for Venus and Earth in Fig. 2. The triangles are mostly scattered in a range between the radial direction and the direction perpendicular to the radial direction in the upper panel of Venus observation. In the lower panel of Earth observation the triangles are gathered in a small range deviating a little from the vertical direction of the spiral to the radial direction. Most of the normal's azimuthal angles are distributed between the radial direction and the direction perpendicular to the spiral, suggesting a tendency for rotation of the normal toward the radial direction. This is consistent with an earlier study by Behannon et al. (1981). The range of normal's elevation at Earth is consistent with an earlier study showing that the sector boundary surfaces at $1 \mathrm{AU}$ are highly inclined $\left(\geq 45^{\circ}\right)$ with respect to the ecliptic plane (Klein and Burlaga, 1980), except one case whose elevation is $70^{\circ}$ (no. 4 in Table 1). At Venus the normals also tend to have their elevation smaller than $\sim 45^{\circ}$, meaning that the current sheet also highly inclined out of the solar equatorial plane. But the normal's elevation angles are distributed in a wider range than those at Earth. There are four normals with elevation angle larger than $60^{\circ}$, indicating a tendency that more current sheets can be more parallel to the solar equatorial plane at Venus. Moreover the distribution of the elevation is relatively flat. There is no strongly preferred orientation among the normals at Venus within the range of $|\theta|<50^{\circ}$.

The larger scatter in the azimuth and elevation of normals at Venus indicates that the current sheet orientation is more variable at $0.72 \mathrm{AU}$ than at $1 \mathrm{AU}$. Examining the magnetic structures inside the selected 32 sector boundaries, we find that more than $50 \%$ (10 cases at Venus and 8 at Earth) contain regions with ambiguous or mixed polarities. For the sector boundaries at Venus of nos. 2, 4, and 15 whose normals have their azimuth deviating much from the vertical of the spiral direction and their elevation larger than $60^{\circ}$, we find that their IMFs inside the whole transition region tend to rotate in the $\mathrm{X}-\mathrm{Y}$ plane intermittently. For these three cases, minimum variance analysis of several shorter intervals including most of the ambiguous or mixed polarities generally give nearly the same results for the normals in elevation but different in azimuth. When the intervals only include the major transitions, different results for the normals both in elevation and azimuth are obtained. The ambiguous or mixed polarity within the transition region may be related to solar conditions, such as complicated magnetohydrodynamic boundary layer between closed and open field lines (Behannon et al., 1981), or small-scale ejections from multiple helmet streamers (Crooker et al., 1993). It can also result from the small-scale structures observed by $\mathrm{SOHO} /$ LASCO to travel along pre-existing streamers (Sheeley et al., 1997; Howard et al., 1997). If the small-scale structures dissipate gradually, it is likely that they affect the current sheet a little more at $0.72 \mathrm{AU}$ than at $1 \mathrm{AU}$. This can explain the larger scatter in the azimuth and elevation of normals at Venus than at Earth.

For the 16 pairs, the scatterplots of the normal's elevation differences versus the Carrington longitude difference and the heliographic latitude difference between Venus and Earth are shown in Fig. 3. Figure 3a indicates that the elevation differences are generally small when the longitude differences between Venus and Earth are small $\left(|\Delta \theta|<30^{\circ}\right.$ for $|\Delta \Phi|<40^{\circ}$, enclosed by dashed lines). Figure $3 \mathrm{a}$ also shows that a large elevation difference has a tendency to be related with large longitude difference, but the reverse is not always true. Thus, the differences of inclination at Earth and Venus observed for $\Delta \Phi>40^{\circ}$ are most likely due to time variation of the helmet streamer during the rotation of the source region with the sun. This view is consistent with the result of recent numerical simulations, which suggest that streamers have a tendency to evaporate and be continually reforming (Suess et al., 1996). But we can have a small difference in $\Delta \theta$ depending on the stability of the inclination even for a large $\Delta \Phi$. This is evidenced by the several triangles at the bottom in Fig. 3a. For these cases, the sector boundaries at both sites are generally well-defined or not disturbed by small-scale structures. The several cases enclosed by dashed lines in Fig. $3 \mathrm{a}$ are also enclosed by dashed lines in Fig. 3b. The
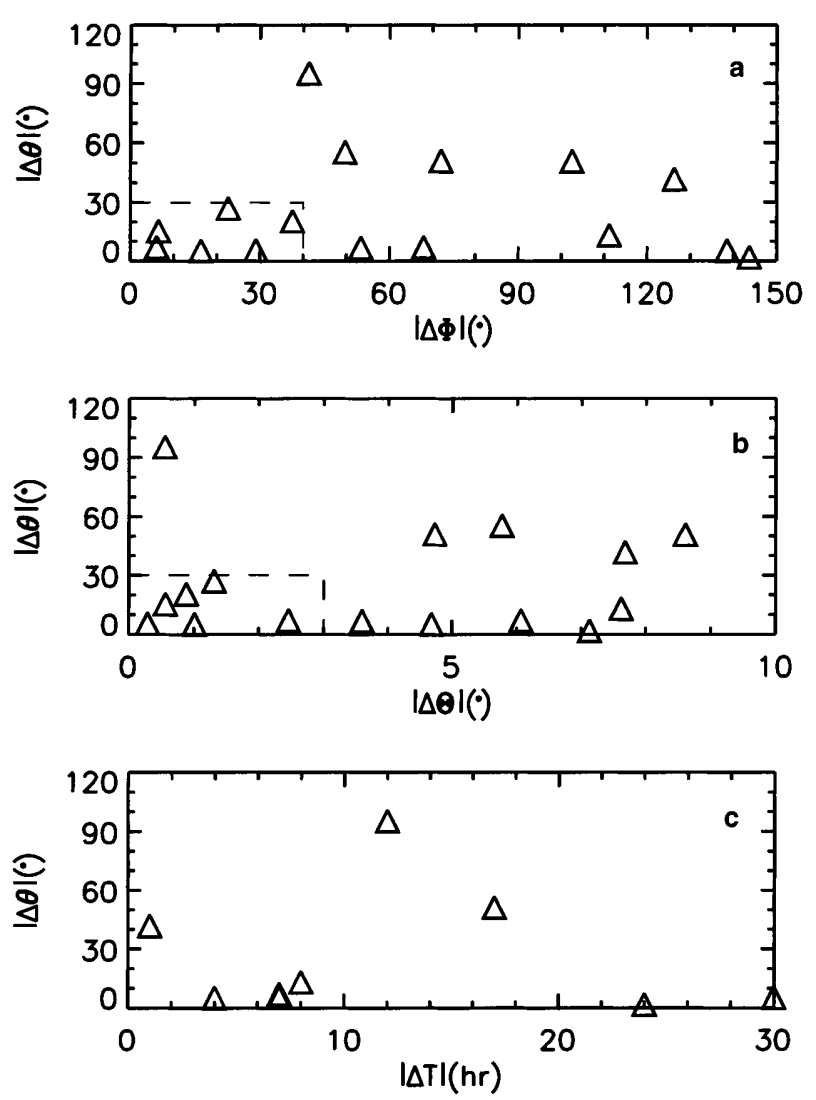

Fig. 3. Scatterplots showing normal's differences $(\Delta \theta)$ versus (a) longitude difference $(\Delta \Phi)$, (b) latitude difference $(\Delta \Theta)$, (c) time lag $(\Delta T)$. Note that there are six triangles gathering at both the lower left corners of $\mathbf{a}$ and $\mathbf{b}$, which are enclosed by dashed lines 
heliographic latitude differences between these pairs are also reasonably small. It can be said that a streamer belt normally maintains a constant sheet inclination typically for three days, corresponding to $40^{\circ}$ solar rotation. The heliospheric current sheet inclination tends to be maintained during propagation of the solar wind from 0.72 to 1 AU. Such a result would be in agreement with the results of Behannon et al. (1981) in their study of sector boundaries over the heliocentric distance range between $0.31 \mathrm{AU}$ and $1 \mathrm{AU}$. They found no radial dependence of the heliospheric current sheet properties (see also Burton, et al., 1994).

Figure $3 \mathrm{c}$ shows a scatterplot of $\Delta \theta$ and $\Delta T$ for the nine pairs with thin $(<3 \mathrm{~h})$ transition regions both at Venus and Earth. It would not be surprising that there are several triangles with both small $\Delta T(<10 \mathrm{~h})$ and $\Delta \theta$, probably resulting from the streamer belts without much temporal variations. A pair of sector boundaries related with a large $\Delta T$ reflect either the geometry, or the temporal variation of the streamer belt. It is also very likely that a large $\Delta T$ indicates the mixed effects of the mentioned above. We concentrate our discussion here on the geometry and the changes of the neutral line position or orientation. If the heliospheric current sheet is inclined with a small angle to the solar equatorial plane and the latitude difference between Venus and Earth is large, $\Delta T$ can be large for a small $\Delta \theta$. Because the heliospheric current sheet in this study is generally highly inclined out of the solar equatorial plane both at Venus and Earth, there is no such cases found here. If the helmet streamer changes position (drifting eastward or westward), $\Delta T$ can also be large for a small $\Delta \theta$. The two triangles at the right bottom are examples of neutral line shift. Of which one case is discussed in section 4.1. As for large $\Delta T$ and $\Delta \theta\left(\geq 30^{\circ}\right)$, such cases occur if we observe the solar wind originated from two sites of a curved neutral line. Consulting the heliospheric current sheet structure from the source surface model (Hoeksema and Scherrer, 1986), neither of the two triangles with large $\Delta \theta$ and $\Delta T$ seems to be caused by a curved neutral line. If the neutral line rotates somehow, $\Delta \theta$ will be large but $\Delta T$ can be either small or large depending on the geometry and history of the streamer belt. This can account for the triangle with large $\Delta \theta$ and small $\Delta T$ (nr. 12 in Table 1), confirmed by the source surface model (Hoeksema and Scherrer, 1986).

\section{Case study}

Some more details are shown about the effects of the temporal variations in solar source regions by two cases. One case suggests the shift of neutral line. The other is about a pair of multiple current sheet crossings at Venus and a single sheet crossing at Earth.

\subsection{Shift of neutral line}

Here we present a pair of sector boundaries with small $\Delta \theta$ and large $\Delta T$ (T5 in Fig. 1 and no. 10 in Table 1), which suggests the shift of neutral line. Fig. 4 shows the IMF vector plots of this pair around 4 Feb. 1981 at Venus and 26 Jan. 1981 at Earth. The coordinates are respectively VSO and GSE. At Venus from 0300 UT, through 1100 UT, 4 Feb. 1981, shown by line V1, the IMFs projected on the $\mathrm{X}-\mathrm{Y}$ plane are parallel to the outward spiral direction and have nearly the same amplitude. Projections on the $\mathrm{X}-\mathrm{Z}$ and $\mathrm{Y}-\mathrm{Z}$ planes vary irregularly because of $B_{z}$ 's variation. At $1200 \mathrm{UT}, 4 \mathrm{Feb}$. 1981, the IMF's component projected on the X-Y plane decays suddenly and remains low until $1600 \mathrm{UT}, 4$ Feb. 1981, as shown by line V2. After 1700 UT, 4 Feb. 1981, as shown by line V3, the IMF is parallel to the inward spiral direction. The time interval used for minimum variance analysis is marked by line VI for Venus and EI for Earth. The latitudes of Venus and Earth when they crossed the sector boundaries were $1.6^{\circ}$ and $-5.6^{\circ}$, respectively. The difference between them was $7.1^{\circ}$. For this pair of sector boundaries, their minimum variance directions (normals to current sheets) are $\left(49.5^{\circ}, 5.1^{\circ}\right)$ and $\left(50.9^{\circ}, 6.9^{\circ}\right)$, as shown in Table 1 . The elevation difference between two normals is only about $1.8^{\circ}$. A sector boundary highly inclined to the ecliptic plane was observed for both Venus and Earth.

Taking into account the sun's rotational effect and the propagation time required for the solar wind to travel from Venus to Earth, we get a positive $\Delta T$ of $24 \mathrm{~h}$, meaning that the sector boundary crossed Venus $24 \mathrm{~h}$ earlier than calculated; in other words, the Earth observation of the sector boundary was $24 \mathrm{~h}$ later than calculated. There are three possible explanations for the large time lag: (1) The streamer belt which generates the
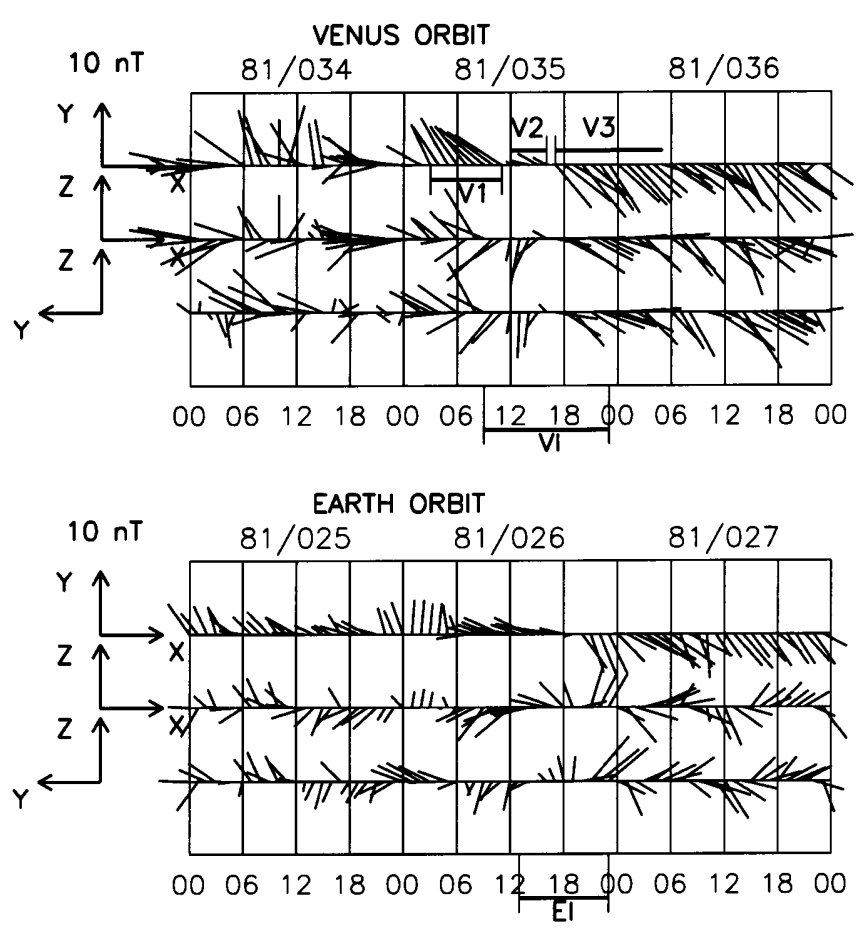

Fig. 4. IMF vector plots for sector boundary pair of no. 10 in Table 1. Single current sheets highly inclined out of the solar equatorial plane are observed both at Venus and Earth. However, a large time lag are found, suggesting the shift of the streamer belt 
sector boundary moved westward. (2) Two separate parts of the same sector boundary were observed at Earth and Venus $\left(24 \mathrm{~h}\right.$ is equivalent to about $13^{\circ}$ longitudinally). (3) The solar wind speed at Venus was much larger than at Earth. Using the speed observed at 1900 UT, 26 Jan. 1981, at Earth, and assuming that the speed is constant from 0 through $1 \mathrm{AU}$, we found that the spiral direction is $139.4^{\circ}$ azimuthally at Venus and $130.0^{\circ}$ azimuthally at Earth. The normal's azimuthal angles, $49.5^{\circ}$ and $50.9^{\circ}$, indicate that the sector boundaries are nearly parallel to the spiral direction. So we can conclude that the difference between the solar wind speeds at Venus and at Earth should be small. Consequently, the third possibility concerning the solar wind speed can be eliminated. Considering the current sheet structure computed at the source surface for Carrington rotation 1704 (Hoeksema and Scherrer, 1986), around the solar equatorial plane the neutral line is nearly a straight line, and it inclines in such a way that $\Delta T$ would be negative. This implies that the second possibility can not be true either. Thus, the only possibility is that the streamer belt shifted westward about $13^{\circ}$ in longitude within 9 days. This is confirmed by comparing the source surface model results of CR1704 with those of CR1705 (Hoeksema and Scherrer, 1986).

\subsection{Multiple current sheets crossing}

A pair with a multiple current sheets crossing at Venus and a single sheet at Earth (no. 5 in Table 1) is shown in Fig. 5. Unlike the IMF structures shown in Fig. 4, the IMFs around the sector boundaries here do not change
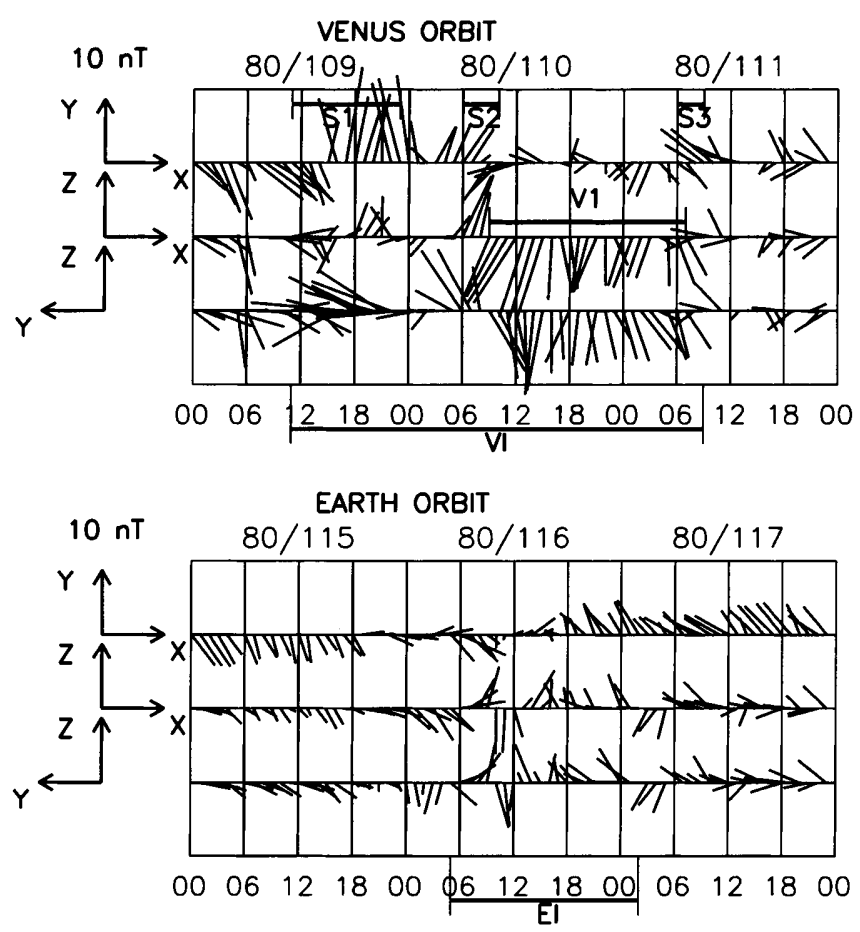

Fig. 5. IMF vector plots for sector boundary pair of no. 5 in Table 1 . A multiple current sheets crossing is observed at Venus (upper panel), while a single current sheet is observed at Earth (lower panel) their direction abruptly. This is especially true for the IMFs observed at Venus. Because of mixed polarities, it is an example of multiple sheets crossing, marked as S1, $\mathrm{S} 2$, and S3. The normal is $\left(20.4^{\circ},-32.7^{\circ}\right)$ for the whole transition region shown by line VI. For the period from 0900 UT, 19 Apr through 0700 UT, 20 Apr 1980, marked by line V1, the IMFs have much larger components in the $\mathrm{Z}$ direction than in the $\mathrm{X}$ and $\mathrm{Y}$ directions. It suggests that this sector boundary contains transient structures, possibly like a small-scale cloud. Applying the minimum variance analysis to the observation at Venus over different intervals, the results of normals are listed in Table 2. Resulting from the transient feature of the sector boundary, it is not surprising that normals from the different intervals within the whole transition region (VI) are different from each other, the same as in Crooker et al. (1993). The two normals from S1 and VI are almost in the same direction. This coincidence seems to imply that the multiple current sheet structure was pushed aside because of the bulge of the small-scale cloud (Crooker et al., 1993). Only a single current sheet with a normal of $\left(45.2^{\circ},-12.3^{\circ}\right)$ was observed at Earth. Although containing ambiguous polarities, the transition is comparatively simple, from 0500 UT, 25 Apr 1980, through 0200 UT, 26 Apr 1980, as shown by line EI. And the IMF magnitude in the interior of the sector boundary at Earth is much smaller than that at Venus. The streamer dynamics lead to a sector boundary with multiple current sheets observed at Venus, and a single current sheet observed at Earth. The elevation difference between the normals to VI and EI is about $20^{\circ}$, suggesting that the effects of small-scale structures are not very strong on the inclination of the sector boundary. This case study support the new view proposed by Crooker et al. (1993) that the sector boundary acts as a conduit for small-scale ejections.

\section{Conclusion}

We compared the IMF polarities at Venus and Earth. The sector structures basically maintain the same from 0.72 to $1 \mathrm{AU}$. However the fine structures at the sector transition are rather complicated. Transient signatures often occurred at the locations of sector boundaries in the observations from December 1978 through May 1982, implying the dynamic nature of the sector boundary. This result suggests that transient structures were often ejected from the coronal streamer belt around the solar maximum.

Table 2. MVA results from the multiple current sheets at Venus of no. 5 in Table 1. The coordinate of normals here is VSO

\begin{tabular}{lll}
\hline & Interval & Normal \\
\hline S1 & $80 / 109 / 11-80 / 109 / 23$ & $(19.5,-27.4)$ \\
S2 & $80 / 110 / 06-80 / 110 / 10$ & $(-23.7,-9.8)$ \\
S3 & $80 / 111 / 06-80 / 111 / 09$ & $(34.7,30.5)$ \\
VI & $80 / 109 / 11-80 / 111 / 09$ & $(19.7,-29.1)$ \\
\hline
\end{tabular}


For the selected 16 pairs of sector boundaries, their inclinations were determined by using minimum variance analysis. Most of the normals to the current sheet have their azimuth distributed between the radial direction and the direction perpendicular to the spiral direction. The normals' elevations tend to be smaller than $\sim 45^{\circ}$, meaning that the heliospheric current sheet is highly inclined out of the solar equatorial plane, in particular at Earth.

The scatter in the azimuth and elevation of normals is larger at Venus than at Earth. Examining the magnetic structure within the sector boundaries, we find that more than $50 \%$ are related with small-scale structures. This is especially true for sector boundaries at Venus. If the small-scale structures dissipate gradually during propagation away from the sun, it is very likely that they affect the current sheet a little more at $0.72 \mathrm{AU}$ than at $1 \mathrm{AU}$. This can explain the larger scatter in the azimuth and elevation of normals to the current sheet at Venus than at Earth.

When the longitude difference between Venus and Earth is small $\left(<40^{\circ}\right.$ longitudinally), the inclinations of the sector boundaries at Venus and Earth have been found to be close to each other $\left(\Delta \theta<30^{\circ}\right)$, especially for sector boundary pairs without small-scale structures. This indicates that the heliospheric current sheet inclination tends to be maintained during propagation of the solar wind from $0.72 \mathrm{AU}$ to $1 \mathrm{AU}$, and the lifetime of a streamer belt for maintaining a constant sheet inclination is about 3 days. The large difference $\left(\Delta \theta>30^{\circ}\right)$ between the inclinations at Venus and Earth for $\Delta \Phi>40^{\circ}$ is most likely due to the change of the neutral line orientation during the rotation of the source region with the sun.

Finally, two case studies are presented. One case is a pair of sector boundaries with small $\Delta \theta$ and large $\Delta T$, suggesting the shift of the neutral line. The other is a pair with a multiple current sheets crossing at Venus and a single sheet at Earth, which supports the suggestion that the sector boundary acts as a conduit for smallscale ejections (Crooker et al., 1993).

Acknowledgements. This work was done when one of the authors (GM) held a fellowship funded by the Science and Technology Agency of Japan. The solar wind data used in this study were supplied by the US National Space Science Data Center, NASA, Goddard Space Flight Center, through the Space Observation Data Center, ISAS, Japan. The authors are indebted to both these centers for their support. GM wishes to thank K. Hocke and J.L. Wang for their helpful comments on the manuscript.

Topical Editor R. Schwenn thanks N. V. Crooker and S. T. Suess for their help in evaluating this paper.

\section{References}

Alfvén, H., Electric currents in cosmic plasma, Rev. Geophys., 15, 271-284, 1977.

Behannon, K. W., F. M. Neubauer, and H. Barnstorf, Fine-scale characteristics of interplanetary sector boundaries, J. Geophys. Res., 86, 3273-3287, 1981.
Behannon, K. W., L. F. Burlaga, and A. J. Hundhausen, A comparison of coronal and interplanetary current sheet inclinations, J. Geophys. Res., 88, 7837-7842, 1983.

Burton, M. E., N. U. Crooker, G. L. Siscoe, and E. J. Smith, A test of source-surface model predictions of heliospheric current sheet inclination, J. Geophys. Res., 99, 1-9, 1994.

Couzens, D. A., and J. H. King, Interplanetary Medium Data Book, Supplement 3, 1977-1985, NSSDC/WDC-A R\&S, 86-04, NASA/GSFC, 1986

Crooker, N. U., G. L. Siscoe, S. Shodhan, D. F. Webb, J. T. Gosling, and E.J. Smith, Multiple heliospheric current sheets and coronal streamer belt dynamics, J. Geophys. Res., 98, 93719381, 1993.

Hoeksema, J. T., and P. H. Scherrer, The solar magnetic field-1976 through 1985, Rep. UAG-94, World Data Cent. A for Sol. Terr. Phys., Boulder, Colo., 1986.

Howard, R. A., G. E. Brueckner, O. C. St. Cyr, D. A. Biesecker, K. P. Dere, M. J. Koomen, C. M. Korendyke, P. L. Lamy, A. Llebaria, M. V. Bout, D. J. Michels, J. D. Moses, S. E. Paswaters, S. P. Plunkett, R. Schwenn, G. M. Simnett, D. G. Socker, S. J. Tappin, and D. Wang, Observations of CMEs from SOHO/LASCO, in Coronal Mass Ejections, Geophys. Monogr. Ser., Vol 99, edited by N.U. Crooker, J.A. Joselyn, and J. Feynman, 17-26, AGU, Washington, D.C., 1997.

Kahler, S. W., and R. P. Lin, An examination of directional discontinuities and magnetic polarity changes around interplanetary sector boundaries using E $>2 \mathrm{KeV}$ electrons, Solar Phys., 161, 183-195, 1995.

Klein, L., and L. F. Burlaga, Interplanetary sector boundaries 19711973, J. Geophys. Res., 85, 2269-2276, 1980.

Klein, L., and L. F. Burlaga, Interplanetary magnetic clouds at 1 AU, J. Geophys. Res., 87, 613-624, 1982.

Lepping, R. P., and K. W. Behannon, Magnetic field directional discontinuities: 1. minimum variance errors, J. Geophys. Res., 85, 4695-4703, 1980.

Marubashi, K., Corotating and transient structures of the interplanetary magnetic fields at Venus and Earth, Solar Wind Eight, edited by D. Winterhalter, J. T. Gosling, S. R. Habbal, W. S. Kurth, and M. Neugebauer, AIP Conf. Proc. 382, 522, 1996

Russell, C. T., R. C. Snare, J. D. Means, and R. C. Elphic, Pioneer Venus orbiter fluxgate magnetometer, it IEEE Trans. Geosci. Remote Sens., GE-18, 32-35, 1980.

Schulz, M., Interplanetary structure and the heliomagnetic equator, Astrophys. Space Sci., 24, 371, 1973.

Sheeley, N. R., Jr., Jr, Y.-M. Wang, S. H. hawley, G. E. Brueckner, K. P. Dere, R. A. Howard, M. j. Koomen, C. M. Korendyke, D. J. Michels, S. E. Paswaters, D. G. Socker, O. C. St. Cyr, D. Wang, P. L. Lamy, A. Llebaria, R. Schwenn, G. M. Simnett, S. Plunkett, and D. A. Biesecker, Measurements of flow speeds in the corona between 2 and 30 Rs, Ap. J., 484, 472-478, 1997.

Sonnerup, B. U. O., and L. J. Cahill, Jr., Magnetopause structure and attitude from Explorer 12 observations, J. Geophys. Res., 72, 171-183, 1967.

Suess, S. T., Comment on "A Comparison of Coronal and Interplanetary Current Sheet Inclinations" by K. W. Behannon, L. F. Burlaga, and A. J. Hundhausen, J. Geophys. Res., 89, 11059-11060, 1984.

Suess, S. T., D. J. McComas, and J. T. Hoeksema, Prediction of the heliospheric current sheet tilt: 1992-1996, Geophys. Res. Lett., 20, 161-164, 1993.

Suess, S. T., A.-H. Wang, and S. T. Wu, Volumetric heating in coronal streamers, J. Geophys. Res., 101, 19957-19966, 1996.

Villante, U., and R. Bruno, Structure of current sheets in the sector boundaries: Helios 2 observations during early 1976, $J$. Geophys. Res., 87, 607-612, 1982. 